\title{
Design and biomechanical performance analysis of a user-friendly orthotic device
}

\author{
Muhammad Iftekharul Rakib ${ }^{a}$, Imtiaz Ahmed Choudhury ${ }^{\text {a,* }}$, Sajjad Hussain ${ }^{\text {b }}$, Noor Azuan Abu Osman ${ }^{\text {b }}$ \\ ${ }^{a}$ Manufacturing System Integration (MSI), Department of Mechanical Engineering, Faculty of Engineering, University of Malaya, 50603 Kuala Lumpur, Malaysia. \\ ${ }^{\mathrm{b}}$ Center for Applied Biomechanics (CAB), Dept. of Biomedical Engineering, Faculty of Engineering, University of Malaya, 50603 Kuala Lumpur, Malaysia.
}

A R T I C L E I N F O

Article history:

Received 29 May 2014

Accepted 29 September 2014

Available online 6 October 2014

\section{Keywords:}

Design

Stance-control-orthosis

Finite-element-analysis

Weight optimization

Gait cycle

\begin{abstract}
A B S T R A C T
Material selection and proper design are essential for developing a new product, especially biomedical devices. Stance-control-orthosis (SCO) are walking assistive device for the patients with weak quadriceps. This manuscript presents the development of a prefabricated SCO with suitable material and design. It is $45 \%$ lighter than commercially available prefabricated device. It contains some adjustable features to make it competent for wide range of patients in terms of height $(153-183 \mathrm{~cm})$ and weight. Finite element analysis (FEA) was used for selecting the best material and optimizing weight of different components of this device. Basic structures the device were made of aluminum alloy 7075-T6. Carbon-fiber epoxy composite material was used for fabricating the support braces of the device and a polypropylene made foot part was included. Components were fabricated according to FEA results and had been evaluated by mechanical tests. Almost similar results of FEA were found during mechanical tests. The device gait performance was assessed and compared with commercially available prefabricated SCO. The results revealed a better gait. Proper material selection and design made this orthotic device light, structurally stable, functionally suitable and cosmetically acceptable to users.
\end{abstract}

2014 Elsevier Ltd. All rights reserved.

\section{Introduction}

Every year many individuals sustain quadriceps weakness or knee instability because of paralysis, spinal cord injury, or polio. They are usually prescribed KAFO, which provides knee stability and protects the knee from collapsing during the standing and stance phase of walking. But it keeps the knee in full extension throughout the gait cycle. The human gait cycle can be described as Fig. 1. Since KAFO resists knee flexion during swing phase thus it causes abnormal gait pattern. This unnatural gait pattern includes hip hiking of the braced leg, circumduction, contralateral foot vaulting and higher metabolic energy expenditure [1-3]. It also reduces $23-33 \%$ of gait efficiency of a patient and elevate center of mass position $65 \%$ vertically [4]. Therefore, the rate of rejection is very high (58-79\%) $[5,6]$. KAFOs limit the ability of users to walk on irregular or inclined surfaces, ascend or descend stairs, and step over obstacles because of inadequate toe clearance. Moreover, its abnormal gait pattern causes pain and the joint dis-function of the hip and lower back [1].

\section{* Corresponding author. Tel.: +60 379675384 .}

E-mail addresses: muhd.rakib@outlook.com (M.I. Rakib), imtiaz@um.edu.my (I. A. Choudhury), sajjadhussain@um.edu.my (S. Hussain), azuan@um.edu.my (N.A.A. Osman).

http://dx.doi.org/10.1016/j.matdes.2014.09.075 0261-3069/○ 2014 Elsevier Ltd. All rights reserved.
SCOs are designed to keep the knee locked during stance phase and allow knee flexion during swing phase. Studies revealed that SCOs improve gait efficiency and kinematics, enhance knee stability, walking velocity and stride length, reduce energy requirement and oxygen consumption of walking, and allow more normal gait. SCOs also allow stair ascent or descent and ambulation on inclined surface $[1,5,8-16]$.

The success of commercially viable prefabricated SCO is limited because of weight, bulkiness, lack of adequate cosmetic appeal, and cost [10]. This article presents an optimized design with finite element analysis (FEA), material selection, mechanical testing, and biomechanical performance evaluation of a prefabricated SCO device. In this research work the efforts had been made to overcome the challenges for achieving greater user acceptance and lower the rejection rate. The weight of the device was optimized with FEA output and the computer-based database of material selection system. Three different materials such as carbon-fiberepoxy composite material, aluminum alloy 7075-T6 and polypropylene were used for making the device lighter and sufficiently strong to meet the purposes of the device. The FEA results were evaluated and verified by mechanical tests. Design of this device is simple and eliminates the manufacturing complexity that leads lower production cost. Moreover, its off-the-shelf design and adjustable features make it usable for wide range of patients. 


\section{Materials and methods}

\subsection{Device description}

The device was a prefabricated and adjustable SCO (Fig. 2a). The device design was modular, compact and light. The thigh and shank cuffs were made of carbon-fiber-epoxy composite material. Side bars were made of aluminum-alloy 7075-T6 and the foot section was made of polypropylene. Commercially available stancecontrol-knee-joint (SCKJ) and double-action-ankle-joint of Becker Orthopedics were used. The aluminum-alloy made side bars contained adjustable feature, therefore, the overall height of the device could be easily adjusted by changing the position of the bolts (Fig. 2b). Thigh and shank cuffs contained adjustable slots (Fig. 2c and d). Cuffs were attached with the side bars by bolts and easily adjustable by changing the bolts position through the slots. These all adjustable features made it suitable to accommodate patients from $153 \mathrm{~cm}$ to $183 \mathrm{~cm}$ in height. The SCKJ locking and unlocking system was control cable operated. The SCKJ remained locked throughout the stance phase. During the end of stance phase, maximum ankle dorsiflexion took place and caused the control cable to pull the lever of SCKJ and unlocked it. SCKJ remained unlocked throughout the swing phase. Before the heel strike, the knee extension caused the lever to reengage the lock of SCKJ. The overall weight of the device was only $1.1 \mathrm{~kg}$. It offered three modes of operation; locked mode in entire gait cycle, stance control mode and free knee motion mode.

\subsection{Components design and tests}

The components design and tests can be described as in Fig. 3.

2.2.1. Thigh and shank cuffs

2.2.1.1. CAD works and FEA. The commercially available thigh and shank adjustable cuffs are made of mild steel. Recently, the use of composite materials emerges promisingly in the biomedical products and devices. In SolidWorks 2013 (2013Dassault Systems SolidWorks Corp.), 3D models of a thigh and a shank cuff were created. Computed tomography (CT) scan images of nine patients (five male and four female) were reconstructed with MIMICS software (Materialise, Belgium) for this purpose. The external profile of leg of those patients were constructed from these CT data. The age, height, and weight of these patients were different ( $33 \pm 6$ years, $167 \pm 15 \mathrm{~cm}$ and $71 \pm 16 \mathrm{~kg}$ ). The CT scan data of these nine patients were considered in order to create the 3D models of prefabricated cuffs those would be off-the-shelf in size. So that, the cuff models became suitable for wide range of patients with the adjustable features. Hence, all the geometric parameters for shape and size of cuff models were taken by analyzing the external profile of those patient's leg.

The purpose of this FEA was to develop prefabricated cuffs with carbon-fiber-composite materials which could be better substitute of commercially available mild steel cuffs. The new cuffs were lighter than the mild steel cuffs with similar structural strength. The thickness of the composite cuffs were optimized in order to make it lighter and user-friendly. The methodology can be described as below:

- The yield loads of mild steel cuffs were considered for FEA in order to develop light-weight cuffs possessing a similar or better strength than the commercially available mild steel cuffs.

- Yield loads of the commercially available mild steel cuffs were determined by an Instron 3382 Floor Model Universal Testing System. The load was applied as shown in Fig. 4. Deflections due to loading were monitored.

- Properties of the developed carbon-fiber-composite materials were determined by the tensile test in Instron 3382 Floor Model Universal Testing System. Those properties were summarized in Table 1.

- The cuffs were modelled by choosing the material as carbonfiber-epoxy composite material. The properties mentioned in Table 1 were utilized for the analysis.

- The thickness of composite cuffs models were optimized based on the stress formation under the yield load of mild steel cuffs. The thickness of thigh composite cuff was optimized under the yield load of mild steel thigh cuff. Similarly, the thickness of shank composite cuff was optimized under the yield load of mild steel shank cuff. Initially the thickness of the 3D cuff models was considered as $6.00 \mathrm{~mm}$. Loads were applied directly along the adjustable bolt joints, and the opposite side of loading was kept fixed, as shown in Fig. 5. Gradually the thickness of the plies of composite cuffs were reduced and developed stress were monitored. The thickness was reduced until the developed von Mises stress in the 3D cuff models went beyond the allowable stress limit (Tables 2 and 3). The maximum allowable stress was the yield strength of composite material (1490 MPa), mentioned in Table 1.

- Subsequently, the composite cuffs were again modelled with the optimized thickness that found in the above mentioned step. Probable yield loads of the composite cuffs were determined by increasing the applied loads. The loading directions and conditions were also similar as above.

2.2.1.2. Yield load tests. One thigh and one shank cuffs were fabricated with the carbon-fiber-epoxy composite materials according to the results of FEA. All the geometric parameters of the fabricated composite cuffs were followed by the 3D model and the thickness of the cuffs was considered as the optimized thickness found in FEA. The yield loads of these composite cuffs were determined by an Instron 3382 Floor Model Universal Testing System. The loads were applied directly along the adjustable bolt joints and deflections were monitored (Fig. 4). The results were then compared with the yield load and stiffness of the mild steel cuffs. The commercially available mild steel cuffs are $4.50 \mathrm{~mm}$ thick.

22.1.3. Fatigue tests. A two steps cyclic test was conducted to determine the longevity of the fabricated new cuffs. Two loads were selected for undergoing 100,000 cycles. (i) Composite cuffs yield load since load required for don, doff and adjustment is lim-
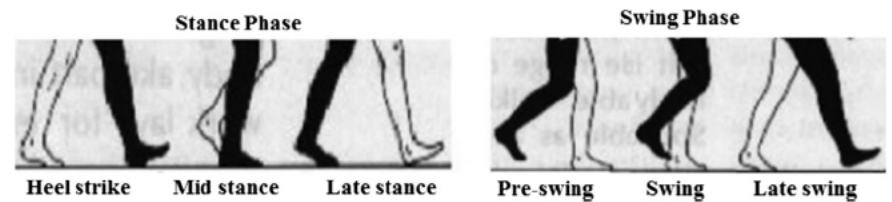

Fig. 1. Human gait cycle [7] 


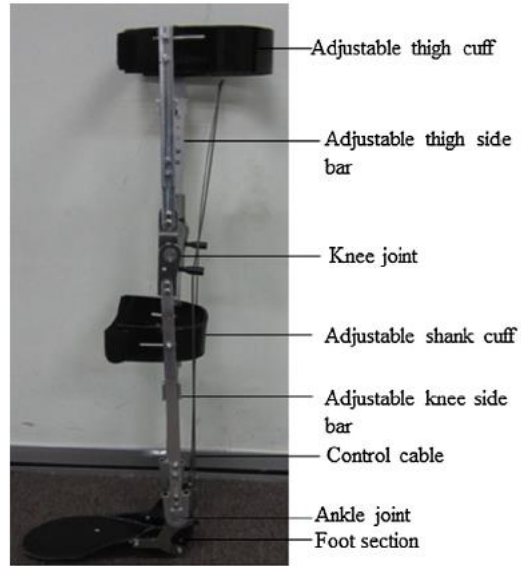

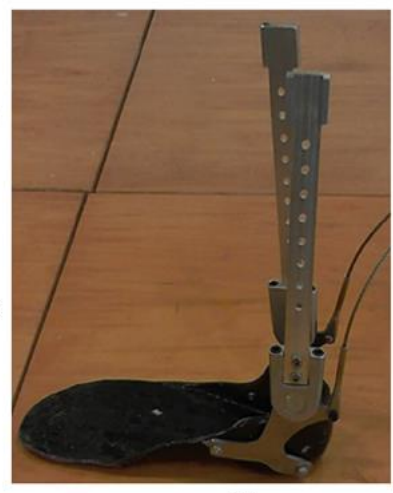

(b)

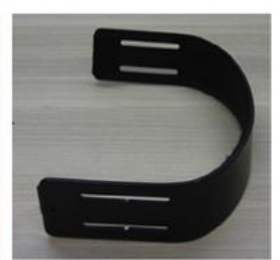

(c)

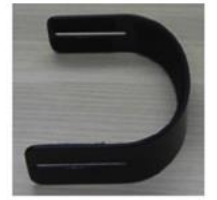

(d)

(a)

Fig. 2. (a) Device, (b) foot section with adjustable knee side bar, (c) adjustable thigh cuff and (d) adjustable shank cuff.

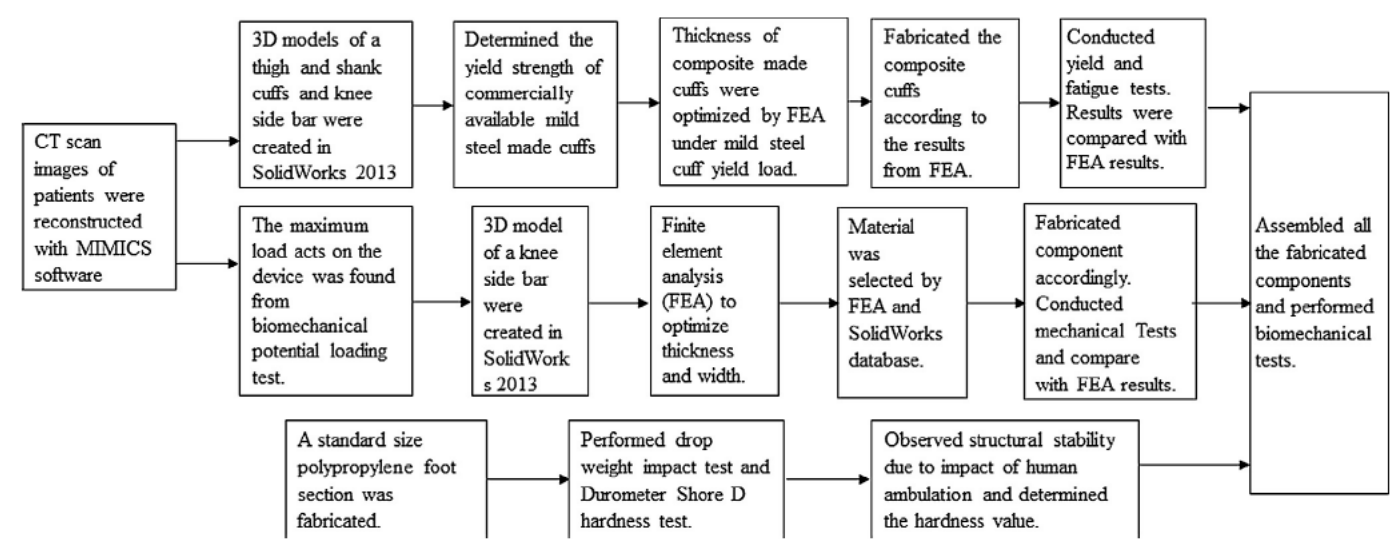

Fig. 3. Steps of components design and tests.

ited within this load and (ii) a load which is $20 \%$ higher than the composite cuffs yield load.

2.2.2. Knee side bars

2.2.2.1. Biomechanical test for potential loading. Three healthy male participants were selected for "potential-loading" test (age $36 \pm 7$ years, height $171 \pm 3 \mathrm{~cm}$ and weight $78.50 \pm 11.50 \mathrm{~kg}$ with orthosis). The maximum load acts on the device was determined by this test while walking with a normal cadence and speed. The ground reaction forces (GRF) at different stages of gait cycle were monitored at $1000 \mathrm{HZ}$ by using a seven camera motion-capturesystem (Vicon Motion Systems; Los Angeles, California), Vicon Nexus 1.8.1 software (Vicon Motion Systems), and force plate (Kistler, Switzerland) in the Gait and Motion Analysis Lab of University of Malaya.

2.2.2.2. CAD works and FEA. During the stance phase of gait cycle, the device supports the patient body weight. The maximum load on the orthotic device was found from the results of "Biomechanical test for potential loading" (Table 6). Firstly, the width and thickness of the knee-side-bar model was optimized by FEA in SolidWorks 2013 considering the maximum load on the device. Factor of safety of the model was determined from the yield strength and maximum stress developed under the considered load. The FEA works can be described as below.

- Initially, a 3D model of adjustable knee-side-bar was created in SolidWorks 2013 and the material was chosen as Aluminum alloy 6061-T6.

- The lower end of the model was kept fixed and axial compressive load was applied from the top, as described in Fig. 7.

- The geometric parameters (width and thickness) were optimized under the maximum load of Table 6 and a minimum allowable factor of safety of 2.50. Stress formation was observed and factor of safety was calculated. Fig. 7(a) shows the stress formation while optimizing the geometric parameters.

- Table 7 describes the optimization procedures. Initially, width and thickness of the knee-side-bar were considered as $25.0 \mathrm{~mm}$ and $6.0 \mathrm{~mm}$ respectively. Loading condition is mentioned above. Stress formation and deflection were observed 


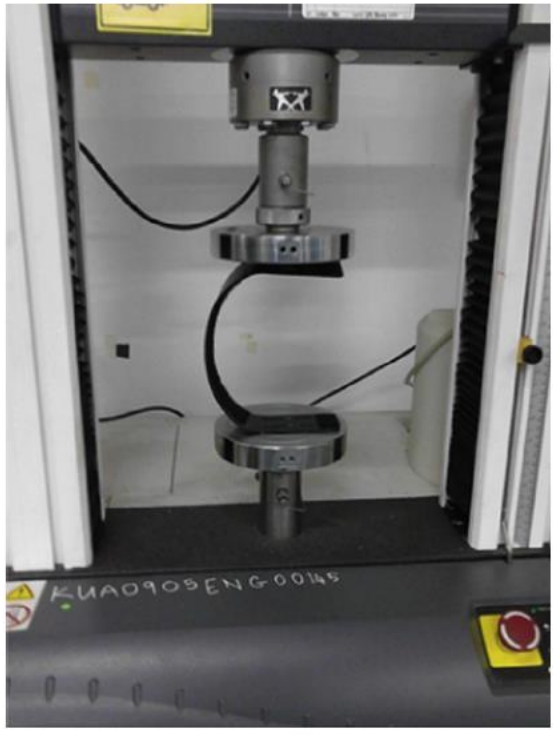

Fig. 4. Yield load test of mild steel and composite made cuffs.

Table 1

Properties of the developed composite material.

\begin{tabular}{ll}
\hline Properties & \\
\hline Average density $\left(\mathrm{kg} / \mathrm{m}^{3}\right)$ & 1560 \\
Elastic modulus in $X$ direction $(\mathrm{GPa})$ & 102.9 \\
Elastic modulus in $Y$ direction $(\mathrm{GPa})$ & 103.2 \\
Elastic modulus in $Z$ direction $(\mathrm{GPa})$ & 138.2 \\
Tensile strength in $Y$ direction $(\mathrm{MPa})$ & 1818 \\
Tensile strength in $Z$ direction $(\mathrm{MPa})$ & 1540 \\
Yield strength (MPa) & 1490 \\
\hline
\end{tabular}

under the maximum load of Table 6. The factor of safety was calculated from the yield strength and maximum stress developed under the considered load. Gradually, the width and thickness were reduced. In every step the stress formation and deflection were observed under the maximum load of Table 6 and factor of safety was calculated. The width and thickness were reduced until the factor of safety in the 3D knee-sidebar model became lower than 2.50 (Table 7).

- After the optimization of geometric parameters, 3D models were created with Aluminum alloy 7075-T6, Aluminum alloy 2014-T6, AISI 1020 carbon steel, and AISI 304 stainless steel. The optimized width and thickness found in the above mentioned steps were utilized for creating these 3D models. Deflections and factor of safety were determined for each model under the maximum load from Table 6. The loading conditions were similar like above.

- Subsequently, the FEA outcomes were compared and suitable material for fabricating the knee-side-bar was selected on the basis of higher safety factor, lighter weight and minimum deflection under load. The database of SolidWorks 2013 was helpful in this aspect. Since the device is prefabricated, therefore, the high factor of safety would make it compatible for different types of patient having diversity in gait pattern. The light-weight and minimum deflection of knee-side-bar would make it comfortable for daily use.
2.2.2.3. Compressive load test. Based on the results from FEA, an adjustable knee-side-bar was fabricated. It was placed in Instron 3382 Floor Model Universal Testing System. Axial compressive loads up to $1000 \mathrm{~N}$ was applied on the knee-side-bar and deflections in respect of applied loads were observed (Fig. 8a).

\subsubsection{Polypropylene made foot section}

A standard size polypropylene made foot section was fabricated; the thickness was $3.0 \mathrm{~mm}$. Polypropylene was selected because of its relatively lower stiffness and lighter properties $[17,18]$. CT scan images of the right leg of nine patients were also used in this aspect [19]. For confirming structural rigidity a drop weight impact test was conducted. A hardness test was also conducted to safeguard the user comfort.

2.2.3.1. Drop weight impact test of foot section. According to Chi et al. [20] the maximum amount of kinematic energy absorbed by a human heel pad during the heel strike phase is $3.99 \mathrm{~J}$ in various condition of walking and running and according to Kövecses et al. [21] it is less than $3.50 \mathrm{~J}$. A drop weight impact test on the foot section was carried out for absorbing a kinematic energy of $4.0 \mathrm{~J}$ during impact. The test was conducted at room temperature with INSTRON CEAST 9310 machine. The foot specimen was clamped with a custom support fixture for footwear provided by Instron. A hemispherical impactor of $16 \mathrm{~mm}$ diameter was dropped on the specimen from a predetermined height. The compressive deflection $(\mathrm{mm})$ and impact force acting time (ms) with respect to energy absorption (J) were observed [22].

22.3.2. Hardness test of foot section. A durometer shore D hardness test of the foot section material was performed in Zwick 3116 hardness tester (Zwick, USA) according to ASTM: D2240. The test specimen was also produced according to ASTM: D2240.

\subsection{Orthosis performance evaluation test}

Three healthy male subjects were selected for the biomechanical performance test of the device. The tests were carried out in the Gait and Motion Analysis Lab of University of Malaya. Three trials of walking over a $7.0 \mathrm{~m}$ walkway inside the gait lab were considered for this test. Every subject walked with three different devices - traditional KAFO, commercially available prefabricated SCO and this new device. The kinematics of the knee joint and hip vertical displacements were observed at $1000 \mathrm{~Hz}$ by using seven camera motion-capture-system (Vicon Motion Systems; Los Angeles, California) and Vicon Nexus 1.8.1 software (Vicon Motion Systems).

\section{Results}

\subsection{Thigh and shank cuffs}

The "yield load test" demonstrated commercially available thigh and shank cuffs yielded at $630 \mathrm{~N}$ and $650 \mathrm{~N}$ load respectively (Fig. 6). The thickness of the composite cuff models were optimized under these respective mild steel cuff load. The optimized thickness was found as $3.0 \mathrm{~mm}$ for composite thigh and shank cuffs. Tables 2 and 3 show the procedures of composite cuffs thickness optimization. Both thigh and shank composite cuffs demonstrated a higher value of developed von Mises stress than the maximum allowable stress, while the thickness was $2.50 \mathrm{~mm}$. Therefore, the $3.00 \mathrm{~mm}$ thickness was selected as optimized thickness for both of the composite cuffs.

The probable yield loads of the composite cuffs having a thickness of $3.0 \mathrm{~mm}$ were also determined by FEA and described in

\section{Link to Full-Text Articles :}


